\title{
The association between physical activity and health-related quality of life among breast cancer survivors
}

\author{
Woo-kyoung Shin ${ }^{1}$, Sihan Song ${ }^{2}$, So-Youn Jung ${ }^{3}$, Eunsook Lee ${ }^{3}$, Zisun Kim ${ }^{4}$, Hyeong-Gon Moon ${ }^{5}$, \\ Dong-Young Noh ${ }^{5}$ and Jung Eun Lee ${ }^{1,6^{*}}$
}

\begin{abstract}
Background: The quality of life for breast cancer survivors has become increasingly important because of their high survival rate and prolonged life expectancy. The purpose of this study was to examine the association of physical activity following diagnosis and health-related quality of life (HRQOL) in breast cancer survivors.
\end{abstract}

Methods: We conducted a cross-sectional study of breast cancer survivors. A total of 231 women aged 21-78 years who had been diagnosed with stages I to III breast cancer and had breast cancer surgery at least 6 months prior were recruited from three hospitals between September 2012 and April 2015 and were included in this study. We asked participants about their HRQOL and engagement in physical activity using structured questionnaires. We examined the association between HRQOL levels and physical activity using a generalized linear model.

Results: Breast cancer survivors in the high physical activity group (3rd tertile) were more likely to have lower scores for fatigue ( $p$ for trend $=0.001$ ) and pain ( $p$ for trend $=0.02$ ) and higher scores for sexual function ( $p$ for trend $=0.007$ ) than those in the low physical activity group (1st tertile). When we stratified participants by stage, we found increasing scores for physical functioning ( $p$ for trend $=0.01$ ) and decreasing scores for fatigue ( $p$ for trend $=0.02$ ) with increasing levels of physical activity in breast cancer survivors with stage I breast cancer. In survivors with stages II and III, we found statistically significant associations with fatigue $(p$ for trend $=0.02)$ and sexual functioning ( $p$ for trend $=0.001$ ).

Conclusions: In conclusion, engagement in physical activity was related to better health-related quality of life among breast cancer survivors. Our findings may warrant further prospective and intervention studies to support the benefit of physical activity in improving the quality of life and survival of Korean breast cancer survivors.

Keywords: Physical activity, Health related quality of life, Breast cancer survivor, Korean women

\section{Background}

Breast cancer was the most common cancer among women in the world in 2012 [1]. Exposure to breast cancer risk factors, increases in early detection, and the development of treatment methods have resulted in a substantial increase in the number of breast cancer survivors. The prognosis for Korean women diagnosed with breast cancer

\footnotetext{
* Correspondence: jungelee@snu.ac.kr

${ }^{1}$ Research Institute of Human Ecology, Seoul National University, Seoul 08826, South Korea

${ }^{6}$ Department of Food and Nutrition, Seoul National University, Seoul 08826, South Korea

Full list of author information is available at the end of the article
}

has also improved, with $91.3 \%$ surviving at least 5 years post-diagnosis in Korea [2].

The majority of breast cancer survivors have mild and moderate levels of physical and psychological treatment side effects, and these can affect their health-related quality of life (HRQOL). Women with breast cancer who received adjuvant chemotherapy tend to experience persistent physical symptoms of cancer and treatment including fatigue $[3,4]$, pain or sensations in the arm or breast [5], hormone-related symptoms [6, 7], and sexual dysfunction $[6,8]$. These symptoms can last for months to even years after completion of cancer treatment and have an adverse effect on their HRQOL $[9,10]$. 
Physical activity is perceived to be an effective nonpharmacological therapy in cancer patients [11, 12] by relieving the distress caused by physical or psychological symptoms. A growing body of evidence supports the idea that increasing physical activity provides important benefits by promoting psychological and physical well-being in cancer patients [12-14]. Recent several studies showed that physical activity had positive effects on physical symptoms, fitness measures, body composition, biological changes such as immune function, psychosocial measures and other multiple aspects of HRQOL [15-21]. However, most of these studies were conducted in Western populations or on only a few survivors in Asia.

Given that research concerning the psychosocial and physical health among breast cancer survivors is important [22], we aimed to examine the association of physical activity following diagnosis and HRQOL in breast cancer survivors.

\section{Methods \\ Study participants}

A total of 307 women aged 21 to 78 years were recruited between September 2012 and November 2015. These women had been diagnosed at three large hospitals in Korea with stage I to III breast cancer according to the American Joint Committee on Cancer (AJCC) criteria and had breast cancer surgery at least 6 months before the baseline. We excluded women with missing medical record $(n=19)$, metastasis after breast cancer diagnosis $(n=17)$, other cancers prior to diagnosis $(n=6)$, or any cancer after diagnosis, but before enrollment $(n=10)$. Women were also excluded if they had implausible levels of physical activity, which corresponded to over the top $1 \%(n=2)$. As a result, our study included a sample of 231 breast cancer survivors.

All procedures for this study were approved by the institutional review board of each hospital. Written informed consent was obtained from all participants.

\section{Assessment of physical activity levels}

Physical activity after breast cancer diagnosis was assessed using a detailed questionnaire. We asked participants about the type, duration and frequency of each physical activity. As additional questions, participants were asked to list up to three types of exercise that they commonly engaged in as well as their duration and frequency. A metabolic equivalent (MET) value was assigned to each activity reported according to the Compendium of Physical Activities [23].

\section{Assessment of health-related quality of life}

We used a Korean version of the European Organization for Research and Treatment of Cancer (EORTC), Quality of Life Questionnaire Core 30 (QLQ-C30) version 3.0 and Quality of Life Questionnaire Breast Cancer Module 23 (QLQ-BR23), both of which have been validated for Koreans after obtaining written permission from the EORTC Study Group [24, 25].

The EORTC QLQ-C30 is a 30-item core-cancer specific questionnaire-integrating system for assessing the HRQOL of cancer patients participating in international clinical trials [26]. The questionnaire incorporates five functional scales (physical, role, emotional, cognitive, and social), three symptom scales (fatigue, nausea and vomiting, and pain), a global health and QOL scale, and single items for the assessment of additional symptoms commonly reported by cancer patients (e.g., dyspnea, insomnia, appetite loss, constipation, and diarrhea), as well as the perceived financial impact of the disease and treatment [23]. All items are scored on 4-point Likert scales, ranging from 1 ("not at all") to 4 ("very much"), with the exception of two items in the global health/ QOL scale which use modified 7-point linear analog scales [26].

The EORTC QLQ-BR23 is a 23-item breast-cancerspecific questionnaire measuring QOL in breast cancer patients. It incorporates four functional scales (body image, sexual functioning, sexual enjoyment, and future perspective) and four symptom scales (systematic therapy side effects, breast symptoms, arm symptoms, and being upset by hair loss). All items are scored on 4-point Likert scales, ranging from 1 ("not at all") to 4 ("very much") [27, 28].

All of the scores from 1 to 4 or from 1 to 7 were converted to a score from 0 to 100 according to the EORTC Scoring manual [26]. A high score for a functional scale represented a high/healthy level of function, a high score for the global health status/QOL represented a high QOL, but a high score for a symptom scale/item represented a high level of symptomatology/problems. A higher score represented a higher ("better") level of function, or a higher ("worse") level of symptoms.

\section{Assessment of demographic, clinical and other lifestyle factors}

The demographic questionnaire captured survivors' dietary supplement use, educational level, marital status, and alcohol intake. We obtained information on survivors' age at diagnosis, height and weight at diagnosis, survival time, AJCC stage at diagnosis, and menopausal status at diagnosis through medical records. We calculated total energy intake based on information on the foods and amounts consumed using 3-day dietary records for each participant. The participants were asked to write down every food and dish they consumed on 3 non-consecutive days, including 2 weekdays and 1 weekend day. 


\section{Statistical analyses}

Categorical data were described as proportions and percentages, and continuous data were described as the mean and standard deviation (SD) for descriptive analysis of characteristics of participants. To compare characteristics, analysis of variance was used for continuous variables, and chi-squared tests for categorical variables. We examined the association between HRQOL level and physical activity using the generalized linear model. The HRQOL level was log-transformed to improve the normality and exponentiated the least square (LS) means. Physical activity levels were grouped into tertiles. Multivariate models included age at diagnosis (years, continuous), energy intake (kcal/day, continuous), dietary supplement use (yes, no), time since surgery (6 months1 year, $1-5$ years, and $\geq 5$ years), AJCC stage at diagnosis (stage I/II/III), education level (high school of less/college or more), marital status (married or cohabitating/ unmarried, divorced or widowed), and the hospital where participants were treated. Statistical significance of interaction terms was estimated by the Wald test, by including a cross-product term of the exposure in the generalized linear model. If we had missing variables of energy intake $(n=26)$, alcohol intake $(n=1)$, education level $(n=3)$, and marital status $(n=1)$, we assigned participants to median or the common category. The statistical software package SAS for Windows version 9.4 was used for all statistical analyses. Statistical significance was determined based on $P$ values $(<0.05)$ and $95 \%$ confidence intervals.

\section{Results}

A total of 231 survivors, aged 21 to 78 years, with stage I, II, or III breast cancer were included in the analysis. Table 1 presents demographic and clinical characteristics according to physical activity levels. Breast cancer survivors of our study had average of 33.66 MET-hours per week. The mean age of the patients was 48.07 years. More than $60 \%$ of the survivors were dietary supplement users (65.37\%), postmenopausal at diagnosis (64.50\%) and married or cohabiting (81.74\%). Approximately $74 \%$ of the survivors were enrolled 1 to 5 years after their surgery and over half of the survivors reported ever drinking alcohol. We did not find significant differences in age, BMI, energy intake, dietary supplement use, time since surgery, AJCC stage, menopausal status, alcohol intake, education level, and marital status according to physical activity levels in breast cancer survivors included in this analysis.

Increasing physical activity was associated with lower scores of fatigue or pain; LS means (95\% CIs) of fatigue for each subsequent tertile were 21.63, 21.00, and 13.30, respectively ( $\mathrm{p}$ for trend $=0.001$ ) (Table 2 ). For pain, LS means $(95 \%$ CIs) for each subsequent tertile were 12.45 , 7.9 , and 6.25, respectively ( $\mathrm{p}$ for trend $=0.02$ ). Breast cancer survivors in the high physical activity group (3rd tertile) were more likely to have higher scores of sexual functioning ( $\mathrm{p}$ for trend $=0.007$ ), than those in the low physical activity group (1st tertile). When we stratified participants by stage, we found increasing scores of physical functioning ( $\mathrm{p}$ for trend $=0.01$ ) and decreasing scores of fatigue ( $p$ for trend $=0.02$ ) with increasing levels of physical activity in breast cancer survivors with stage I (Table 3). Among breast cancer survivors with stage II and III, we found lower scores of fatigue ( $\mathrm{p}$ for trend $=0.02)$, but higher scores of sexual functioning ( $\mathrm{p}$ for trend $=0.001$ ) comparing the 3rd tertile with the 1 st tertitle of physical activity levels (Table 4).

We further examined whether BMI, menopausal status at diagnosis, and time since surgery modified the associations of fatigue, pain, and sexual functioning, all of which reached significance in the main analysis. Scores of fatigue decreased with increasing levels of physical activity in both strata of BMI ( $p$ values for trend $=0.03$ for BMI $<23 \mathrm{~kg} / \mathrm{m}^{2}$ and 0.01 for BMI $\geq 23 \mathrm{~kg} / \mathrm{m}^{2}$ ) (Additional file 1: Table S1). Significant trends were observed for pain among survivors with BMI $\geq 23 \mathrm{~kg} / \mathrm{m}^{2}$ and for sexual functioning among survivors with $B M I<23 \mathrm{~kg} / \mathrm{m}^{2}$, but these interactions were not statistically significant.

Menopausal status at diagnosis did not modify the associations of fatigue, pain and sexual functioning with physical activity (Additional file 1: Table S2). We observed decreasing scores of fatigue with increasing levels of physical activity for both pre- and post-menopausal breast cancer survivors. Although the interaction was not statistically significant, decreasing trends of pain and increasing trends of sexual functioning with increasing levels of physical activity were limited to post-menopausal breast cancer survivors.

We found that fatigue was associated with physical activity levels regardless of time since surgery (Additional file 1: Table S3). Although a decreasing trend for pain and increasing trend for sexual functioning were more evident among survivors who had surgery less than 2 years before the study compared to those who had surgery 2 or more years before the study, these interactions were not statistically significant.

We examined whether age at diagnosis $(<48, \geq 48$ years, median) modified the associations for fatigue, pain, and sexual functioning (Additional file 1: Table S4). We found that decreasing scores of fatigue with increasing levels of physical activity for both age groups, but these interactions were not statistically significant. Significant trend for pain was limited to breast cancer survivors with $\geq 48$ years of age at diagnosis, but to those with $<48$ years of age at diagnosis for sexual functioning, albeit statistically not significant.

\section{Discussion}

We aimed to determine whether physical activity levels after breast cancer diagnosis were related to HRQOL, 
Table 1 Characteristics of study participants according to physical activity levels

\begin{tabular}{|c|c|c|c|c|c|}
\hline & \multicolumn{5}{|c|}{ Physical Activity (MET-hours per week) } \\
\hline & All $(n=231)$ & Tertile $1(n=77)$ & Tertile $2(n=77)$ & Tertile $3(n=77)$ & $P$ value $^{\mathrm{a})}$ \\
\hline Physical activity (METs-hour per week) ${ }^{\mathrm{b}}$ & $33.66(29.86)$ & $7.19(5.29)$ & 26.96 (6.43) & $66.83(27.56)$ & $<0.001$ \\
\hline Age $(\text { year })^{b}$ & $48.07(8.36)$ & $46.94(7.91)$ & $49.16(8.89)$ & $48.12(8.22)$ & 0.31 \\
\hline Body Mass Index $\left(\mathrm{kg} / \mathrm{m}^{2}\right)^{\mathrm{b}}$ & $23.20(3.13)$ & $23.62(3.86)$ & $23.07(2.97)$ & $22.92(2.38)$ & 0.55 \\
\hline Energy intake $(\mathrm{kcal} / \mathrm{day})^{\mathrm{b}}$ & $1778.30(402.76)$ & $1693.07(363.22)$ & $1805.71(398.39)$ & $1825.96(432.12)$ & 0.16 \\
\hline \multicolumn{6}{|l|}{ Dietary supplement use ${ }^{b}$} \\
\hline yes & $151(65.37)$ & $48(62.34)$ & $55(71.43)$ & $48(62.34)$ & \multirow[t]{2}{*}{0.39} \\
\hline no & $80(34.63)$ & $29(37.66)$ & $22(28.57)$ & $29(37.66)$ & \\
\hline \multicolumn{6}{|l|}{ Time since surgery ${ }^{b}$} \\
\hline 6 month - 1 year & $32(13.85)$ & $12(15.58)$ & $8(10.39)$ & $12(15.58)$ & \multirow[t]{3}{*}{0.70} \\
\hline 1 year -5 years & $172(74.46)$ & $58(75.32)$ & $60(77.92)$ & $54(70.13)$ & \\
\hline 5 years $\leq$ & $27(11.69)$ & $7(9.09)$ & $9(11.69)$ & $11(14.29)$ & \\
\hline \multicolumn{6}{|l|}{ AJCC stage $^{\mathrm{b}}$} \\
\hline । & $101(43.72)$ & $32(41.56)$ & $36(46.75)$ & $33(42.86)$ & \multirow[t]{3}{*}{0.90} \\
\hline$\|$ & $108(46.75)$ & $38(49.35)$ & $35(45.45)$ & $35(45.45)$ & \\
\hline III & $22(9.52)$ & $7(9.09)$ & $6(7.79)$ & $9(11.69)$ & \\
\hline \multicolumn{6}{|l|}{ Menopausal status at the diagnosis ${ }^{\mathrm{b}}$} \\
\hline Postmenopause & $149(64.50)$ & $53(68.83)$ & $51(66.23)$ & $45(58.44)$ & \multirow[t]{2}{*}{0.37} \\
\hline Premenopause & $82(35.50)$ & $24(31.17)$ & $26(33.77)$ & $32(41.56)$ & \\
\hline \multicolumn{6}{|l|}{ Alcohol intake $^{b}$} \\
\hline Never drinker & $112(48.70)$ & $36(46.75)$ & $42(54.55)$ & $34(44.74)$ & \multirow[t]{2}{*}{0.44} \\
\hline Ever drinker & $118(51.30)$ & $41(53.25)$ & $35(45.45)$ & $42(55.26)$ & \\
\hline \multicolumn{6}{|l|}{ Education level $^{b}$} \\
\hline High school or less & $126(55.26)$ & $38(49.35)$ & $41(53.95)$ & $47(62.67)$ & \multirow[t]{2}{*}{0.25} \\
\hline College or more & $102(44.74)$ & $39(50.65)$ & $35(46.05)$ & $28(37.33)$ & \\
\hline \multicolumn{6}{|l|}{ Marital status ${ }^{\mathrm{b}}$} \\
\hline Married or cohabitation & $188(81.74)$ & $58(76.32)$ & $61(79.22)$ & $69(89.61)$ & \multirow[t]{2}{*}{0.08} \\
\hline Unmarried or divorced or widowed & $42(18.26)$ & $18(23.68)$ & 16 (20.78) & $8(10.39)$ & \\
\hline
\end{tabular}

MET metabolic equivalent, AJCC American Joint Committee on Cancer

${ }^{a}$ Analysis of variance was used for continuous variables and chi-square test was used for categorical variables

${ }^{b}$ Continuous variables are reported as Mean value (sd) and Categorical variables are reported as No. (\%)

and these associations varied by stage, BMI, menopausal status at diagnosis, time since surgery, and age at diagnosis among breast cancer survivors in Korea. We found that higher physical activity after diagnosis was associated with lower scores of fatigue and pain and higher scores of sexual functioning. When we limited our analysis to stage I or stage II/III, an inverse association for fatigue remained statistically significant in both groups, but physical functioning increased only among survivors with stage I cancer and sexual functioning increased only among survivors with stage II or III cancer, with increasing levels of physical activity.

Consistent with our research, several observational studies found that exercise was linked to improvement of HRQOL. In a prospective study of the Health Eating
Activity and Lifestyle (HEAL) study of breast cancer prognosis, pre- and post-diagnosis recreational physical activity was associated with better physical functioning, and increases in physical activity after cancer diagnosis were associated with less fatigue and pain and better physical functioning in 545 breast cancer survivors [29]. In a large prospective cohort study of breast cancer survivors conducted in Shanghai, women with higher exercise MET scores ( $\geq 8.3$ MET-hours per week) were more likely to have higher scores of total QOL, and the exercise-QOL association remained stable overtime after cancer diagnosis [30]. Other observational studies in Norway [31], Italy [32], the USA [33, 34], and Finland [35] also found that physical activity was associated with improved QOL or less fatigue. 
Table 2 Health-related quality of life (HRQOL) scores according to physical activity levels among breast cancer survivors with stage I to III breast cancer

\begin{tabular}{|c|c|c|c|c|c|}
\hline \multirow[t]{2}{*}{ HRQOL Items } & \multicolumn{5}{|c|}{ Physical Activity (MET-hours per week) } \\
\hline & $\overline{\text { All }}$ & Tertile 1 & Tertile 2 & Tertile 3 & $P$ for trend ${ }^{a}$ \\
\hline \multicolumn{6}{|l|}{ EORTC QLQ-C30, LS means $(95 \%$ Cl) } \\
\hline Global health status / QoL & 206 & $45.25(33.62,60.90)$ & $31.99(23.14,44.22)$ & $40.10(29.17,55.12)$ & 0.64 \\
\hline \multicolumn{6}{|l|}{ Functioning } \\
\hline Physical Functioning & 229 & $78.44(70.20,87.65)$ & $81.12(71.93,91.48)$ & $82.53(73.21,93.05)$ & 0.41 \\
\hline Role Functioning & 230 & $75.68(59.77,95.82)$ & $83.78(64.68,108.51)$ & $90.10(69.98,116.01)$ & 0.18 \\
\hline Emotional Functioning & 231 & $75.16(62.37,90.58)$ & $72.87(59.47,89.31)$ & $75.67(61.95,92.44)$ & 0.89 \\
\hline Cognitive Functioning & 231 & $75.13(63.80,88.47)$ & $72.40(60.60,86.51)$ & $71.47(59.98,85.16)$ & 0.59 \\
\hline Social Functioning & 231 & $56.74(44.62,72.15)$ & $73.08(56.25,94.94)$ & $72.96(56.39,94.39)$ & 0.08 \\
\hline \multicolumn{6}{|l|}{ Symptom } \\
\hline Fatigue & 230 & $21.63(16.07,29.11)$ & $21.00(15.20,29.02)$ & $13.30(9.64,18.36)$ & 0.001 \\
\hline Nausea / Vomiting & 231 & $2.56(1.60,4.09)$ & $2.33(1.40,3.89)$ & $2.54(1.53,4.20)$ & 0.97 \\
\hline Pain & 230 & $12.45(7.44,20.83)$ & $7.90(4.51,13.83)$ & $6.25(3.58,10.93)$ & 0.02 \\
\hline Dyspnea & 228 & $5.83(3.38,10.03)$ & $3.23(1.79,5.85)$ & $4.07(2.25,7.37)$ & 0.35 \\
\hline Insomnia & 229 & $12.60(7.38,21.53)$ & $15.79(8.81,28.29)$ & $16.22(9.07,29.00)$ & 0.42 \\
\hline Appetite loss & 228 & $2.22(1.33,3.70)$ & $2.69(1.54,4.69)$ & $2.10(1.21,3.65)$ & 0.72 \\
\hline Constipation & 229 & $3.69(2.09,6.52)$ & $4.36(2.35,8.09)$ & $3.62(1.95,6.69)$ & 0.86 \\
\hline Diarrhea & 231 & $2.95(1.77,4.91)$ & $2.26(1.30,3.95)$ & $2.35(1.36,4.07)$ & 0.48 \\
\hline Financial Problems & 231 & $4.46(2.56,7.77)$ & $5.96(3.26,10.90)$ & $3.29(1.81,5.95)$ & 0.19 \\
\hline \multicolumn{6}{|c|}{ EORTC QLQ-BR23, LS means $\left(95 \%\right.$ CI) ${ }^{\mathrm{b}}$} \\
\hline \multicolumn{6}{|l|}{ Functioning } \\
\hline Body image & 231 & $36.03(24.05,54.00)$ & $34.95(22.49,54.30)$ & $32.14(20.83,49.58)$ & 0.58 \\
\hline Sexual functioning & 209 & $2.69(1.53,4.71)$ & $2.57(1.43,4.65)$ & $5.34(3.04,9.39)$ & 0.007 \\
\hline Sexual enjoyment & 69 & $16.89(4.80,59.47)$ & $9.65(3.14,29.62)$ & $11.54(3.34,39.90)$ & 0.56 \\
\hline Future perspective & 231 & $17.83(10.65,29.83)$ & $18.04(10.29,31.60)$ & $19.72(11.35,34.24)$ & 0.70 \\
\hline \multicolumn{6}{|l|}{ Symptom } \\
\hline Systematic therapy side effects & 231 & $21.59(16.54,28.19)$ & $23.55(17.62,31.49)$ & $19.06(14.32,25.36)$ & 0.29 \\
\hline Breast symptoms & 231 & $12.45(8.04,19.28)$ & $10.06(6.25,16.20)$ & $10.42(6.52,16.65)$ & 0.52 \\
\hline Arm symptoms & 231 & $18.20(12.41,26.69)$ & $21.04(13.86,31.93)$ & $17.92(11.89,27.02)$ & 0.83 \\
\hline Upset by hair loss & 157 & $17.28(8.92,33.50)$ & $14.78(7.09,30.83)$ & $17.59(8.60,36.00)$ & 0.90 \\
\hline
\end{tabular}

MET metabolic equivalent, EORTC QLQ-C30 European organization for research and treatment of cancer quality of life questionnaire core $30, L S$ least-squares, CI confidence interval, EORTC QLQ-BR23 European organization for research and treatment of cancer quality of life questionnaire breast cancer module 23 ${ }^{\mathrm{a}} \mathrm{P}$ for trend was calculated using the median value of each tertile category as a continuous variable

${ }^{b}$ Adjusted for age (year: continuous), energy intake (kcal/day: continuous), dietary supplement use (yes, no), education level (high school or less, college or more), marital status (married or cohabitated, unmarried or divorced or widowed), time since surgery (6 month - 1 year, 1 year - 5 years, $\geq 5$ years), stage (I, II, or III), and center

Recent meta-analyses of intervention studies reported that exercise reduced fatigue $[11,36]$ and QOL [36] among breast cancer survivors. Recent large randomized clinical trials found that exercise intervention improved cancer survivors' QOL. The Better Exercise Adherence after Treatment for Cancer (BEAT Cancer) study randomized 222 breast cancer survivors who finished primary treatment to a 3-month combined exercise program or usual care and found better QOL at 3 and 6 months in the intervention group than in the usual care group [37]. The Exercise and Nutrition Enhance Recovery and Good Health for You (ENERGY) reported that intensive group-based intervention of diet and physical activity compared to non-intensive attention control improved physical function of HRQOL at 6 and 12 months of intervention, but the difference between intervention and control groups diminished at 24 months [38]. Likewise, a 12-week LIVESTRONG exercise program at the YMCA led to improvement of QOL in 186 cancer survivors (52\% were breast cancer survivors) [39]. 
Table 3 Health-related quality of life (HRQOL) scores according to physical activity levels among breast cancer survivors with stage I breast cancer

\begin{tabular}{|c|c|c|c|c|c|}
\hline \multirow[t]{2}{*}{ HRQOL Items } & \multicolumn{5}{|c|}{ Physical Activity (MET-hours per week) } \\
\hline & $\overline{\text { All }}$ & Tertile 1 & Tertile 2 & Tertile 3 & $P$ for trend ${ }^{a}$ \\
\hline \multicolumn{6}{|l|}{ EORTC QLQ-C30, LS means $(95 \%$ Cl) } \\
\hline Global health status / QoL & 93 & $44.40(27.04,72.90)$ & $33.27(20.51,53.98)$ & $34.72(20.75,58.08)$ & 0.35 \\
\hline \multicolumn{6}{|l|}{ Functioning } \\
\hline Physical Functioning & 101 & $74.97(67.56,83.20)$ & $76.85(69.59,84.87)$ & $84.62(76.17,94.00)$ & 0.01 \\
\hline Role Functioning & 101 & $79.69(60.00,105.84)$ & $71.11(54.25,93.20)$ & $88.10(66.14,117.35)$ & 0.36 \\
\hline Emotional Functioning & 101 & $83.73(57.88,121.12)$ & $79.85(56.16,113.54)$ & $80.11(55.17,116.33)$ & 0.83 \\
\hline Cognitive Functioning & 101 & $77.91(65.43,92.78)$ & $77.49(65.61,91.52)$ & $67.46(56.55,80.48)$ & 0.07 \\
\hline Social Functioning & 101 & $57.30(39.62,82.86)$ & $70.40(49.53,100.07)$ & $76.72(52.85,111.36)$ & 0.12 \\
\hline \multicolumn{6}{|l|}{ Symptom } \\
\hline Fatigue & 101 & $27.95(17.23,45.34)$ & $25.32(15.97,40.16)$ & $16.29(9.99,26.55)$ & 0.02 \\
\hline Nausea / Vomiting & 101 & $2.56(1.15,5.70)$ & $2.75(1.28,5.89)$ & $3.06(1.37,6.87)$ & 0.64 \\
\hline Pain & 101 & $11.16(4.92,25.29)$ & $7.64(3.50,16.65)$ & $5.92(2.59,13.52)$ & 0.12 \\
\hline Dyspnea & 99 & $5.25(2.13,12.96)$ & $3.46(1.46,8.21)$ & $4.02(1.60,10.13)$ & 0.63 \\
\hline Insomnia & 101 & $14.61(6.19,34.49)$ & $14.90(6.57,33.78)$ & $22.14(9.30,52.72)$ & 0.29 \\
\hline Appetite loss & 100 & $1.35(0.54,3.38)$ & $2.04(0.86,4.84)$ & $1.57(0.63,3.91)$ & 0.85 \\
\hline Constipation & 101 & $3.70(1.42,9.67)$ & $6.01(2.41,15.02)$ & $8.35(3.17,22.04)$ & 0.09 \\
\hline Diarrhea & 101 & $3.01(1.36,6.67)$ & $2.27(1.06,4.84)$ & $1.63(0.73,3.65)$ & 0.12 \\
\hline Financial Problems & 101 & $2.82(1.12,7.07)$ & $3.20(1.33,7.69)$ & $1.92(0.76,4.86)$ & 0.33 \\
\hline \multicolumn{6}{|c|}{ EORTC QLQ-BR23, LS means $\left(95 \%\right.$ CI) ${ }^{\mathrm{b}}$} \\
\hline \multicolumn{6}{|l|}{ Functioning } \\
\hline Body image & 101 & $68.77(39.53,119.62)$ & $65.36(38.56,110.80)$ & $57.80(33.04,101.11)$ & 0.51 \\
\hline Sexual functioning & 94 & $4.12(1.54,11.03)$ & $4.56(1.80,11.54)$ & $5.57(2.12,14.60)$ & 0.50 \\
\hline Sexual enjoyment & 33 & $53.86(7.89,367.75)$ & $36.75(7.85,172.11)$ & $21.84(3.57,133.78)$ & 0.25 \\
\hline Future perspective & 101 & $25.27(10.25,62.28)$ & $30.21(12.79,71.39)$ & $29.68(11.93,73.83)$ & 0.75 \\
\hline \multicolumn{6}{|l|}{ Symptom } \\
\hline Systematic therapy side effects & 101 & $16.48(10.39,26.14)$ & $15.77(10.16,24.48)$ & $12.99(8.15,20.70)$ & 0.27 \\
\hline Breast symptoms & 101 & $5.94(3.08,11.44)$ & $6.82(3.65,12.74)$ & $5.27(2.72,10.22)$ & 0.63 \\
\hline Arm symptoms & 101 & $13.47(7.64,23.77)$ & $13.75(8.00,23.62)$ & $11.58(6.52,20.54)$ & 0.55 \\
\hline Upset by hair loss & 69 & $14.10(4.83,41.22)$ & $7.35(2.41,22.41)$ & $15.16(4.30,53.40)$ & 0.80 \\
\hline
\end{tabular}

MET metabolic equivalent, EORTC QLQ-C30 European organization for research and treatment of cancer quality of life questionnaire core 30, LS least-squares, CI confidence interval, EORTC QLQ-BR23 European organization for research and treatment of cancer quality of life questionnaire Breast cancer module 23 ${ }^{\mathrm{a}} \mathrm{P}$ for trend was calculated using the median value of each tertile category as a continuous variable

${ }^{b}$ Adjusted for age (year: continuous), energy intake (kcal/day: continuous), dietary supplement use (yes, no), education level (high school or less, college or more), marital status (married or cohabitated, unmarried or divorced or widowed), time since surgery ( 6 month -1 year, 1 year -5 years, $\geq 5$ years), and center

Small intervention studies of 40 to 59 Korean breast cancer survivors also reported that exercise was beneficial for the reduction of fatigue $[40,41]$ and appetite loss [42] and increases in emotional [41] and physical function [42]. A 12-week mobile application-based exercise intervention improved HRQOL in Korean breast cancer patients [43]. In a randomized trial of 277 breast and colorectal cancer patients (168 with breast cancer), the provision of an exercise motivation package including exercise DVDs, a pedometer, an exercise diary, and an exercise education session increased the role of physical function and decreased diarrhea [44]. A pretest-posttest intervention study suggested that the intervention group with print materials and pedometers showed significantly improved QOL and reduced fatigue compared to standard recommendation group [45].

Several studies have reported that exercise therapy for cancer patients during radiotherapy reduced fatigue, but the reason is not clear. An intervention study of 66 male prostate cancer patients suggested that improvements in physical function by exercise therapy may help overcome radiation fatigue [46]. There is scientific evidence that 
Table 4 Health-related quality of life (HRQOL) scores according to physical activity levels among breast cancer survivors with stage II and III breast cancer

\begin{tabular}{|c|c|c|c|c|c|}
\hline \multirow[t]{2}{*}{ HRQOL Items } & \multicolumn{5}{|c|}{ Physical Activity (MET-hours per week) } \\
\hline & $\overline{\text { All }}$ & Tertile 1 & Tertile 2 & Tertile 3 & $P$ for trend ${ }^{a}$ \\
\hline \multicolumn{6}{|l|}{ EORTC QLQ-C30, LS means $(95 \%$ CI) } \\
\hline Global health status / QoL & 113 & $48.33(32.27,72.39)$ & $31.37(19.83,49.61)$ & $43.56(28.54,66.49)$ & 0.89 \\
\hline \multicolumn{6}{|l|}{ Functioning } \\
\hline Physical Functioning & 128 & $81.46(68.05,97.51)$ & $85.19(69.31,104.72)$ & $81.99(67.23,99.98)$ & 0.98 \\
\hline Role Functioning & 129 & $76.34(53.02,109.91)$ & $100.65(65.83,153.89)$ & $95.71(64.41,142.23)$ & 0.36 \\
\hline Emotional Functioning & 130 & $72.94(59.76,89.03)$ & $67.19(53.42,84.51)$ & $75.44(60.73,93.71)$ & 0.63 \\
\hline Cognitive Functioning & 130 & $77.05(59.64,99.56)$ & $69.47(51.73,93.29)$ & $75.00(56.74,99.13)$ & 0.96 \\
\hline Social Functioning & 130 & $59.44(42.46,83.22)$ & $78.02(52.98,114.91)$ & $70.92(49.17,102.29)$ & 0.49 \\
\hline \multicolumn{6}{|l|}{ Symptom } \\
\hline Fatigue & 129 & $18.19(12.29,26.91)$ & $19.25(12.25,30.25)$ & $11.47(7.43,17.71)$ & 0.02 \\
\hline Nausea / Vomiting & 130 & $2.53(1.38,4.63)$ & $2.20(1.09,4.41)$ & $2.17(1.12,4.19)$ & 0.69 \\
\hline Pain & 129 & $11.40(5.74,22.67)$ & $7.32(3.32,16.17)$ & $6.15(2.87,13.17)$ & 0.14 \\
\hline Dyspnea & 129 & $5.29(2.60,10.74)$ & $2.90(1.28,6.57)$ & $3.93(1.79,8.61)$ & 0.63 \\
\hline Insomnia & 128 & $12.16(6.05,24.45)$ & $19.57(8.75,43.75)$ & $12.05(5.56,26.12)$ & 0.78 \\
\hline Appetite loss & 128 & $2.28(1.25,4.15)$ & $3.06(1.53,6.09)$ & $2.11(1.09,4.10)$ & 0.67 \\
\hline Constipation & 128 & $3.90(1.87,8.15)$ & $3.56(1.53,8.27)$ & $1.93(0.86,4.34)$ & 0.08 \\
\hline Diarrhea & 130 & $2.72(1.36,5.45)$ & $2.11(0.95,4.69)$ & $3.51(1.65,7.46)$ & 0.40 \\
\hline Financial Problems & 130 & $5.33(2.56,11.10)$ & $8.65(3.72,20.11)$ & $4.30(1.94,9.56)$ & 0.42 \\
\hline \multicolumn{6}{|l|}{ EORTC QLQ-BR23, LS means $(95 \% \text { Cl) })^{\text {b }}$} \\
\hline \multicolumn{6}{|l|}{ Functioning } \\
\hline Body image & 130 & $26.17(14.74,46.48)$ & $24.74(12.78,47.91)$ & $22.67(12.13,42.35)$ & 0.66 \\
\hline Sexual functioning & 115 & $1.97(1.00,3.85)$ & $1.61(0.76,3.41)$ & $5.77(2.92,11.39)$ & 0.001 \\
\hline Sexual enjoyment & 36 & $10.24(1.60,65.60)$ & $3.36(0.64,17.61)$ & $7.46(1.29,43.12)$ & 0.97 \\
\hline Future perspective & 130 & $16.23(8.49,31.02)$ & $12.63(5.99,26.62)$ & $15.43(7.62,31.23)$ & $>0.99$ \\
\hline \multicolumn{6}{|l|}{ Symptom } \\
\hline Systematic therapy side effects & 130 & $23.04(16.92,31.36)$ & $30.31(21.25,43.22)$ & $23.11(16.52,32.33)$ & 0.75 \\
\hline Breast symptoms & 130 & $16.84(9.26,30.60)$ & $11.09(5.58,22.06)$ & $14.16(7.39,27.13)$ & 0.77 \\
\hline Arm symptoms & 130 & $19.83(11.69,33.65)$ & $26.64(14.50,48.95)$ & $22.71(12.77,40.37)$ & 0.78 \\
\hline Upset by hair loss & 88 & $19.92(7.97,49.81)$ & $26.82(9.60,74.98)$ & $22.28(8.72,56.92)$ & 0.89 \\
\hline
\end{tabular}

MET metabolic equivalent, EORTC QLQ-C30 European organization for research and treatment of cancer quality of life questionnaire core 30, LS least-squares, CI confidence interval, EORTC QLQ-BR23 European organization for research and treatment of cancer quality of life questionnaire breast cancer module 23 ${ }^{\mathrm{a}} \mathrm{P}$ for trend was calculated using the median value of each tertile category as a continuous variable

${ }^{b}$ Adjusted for age (year: continuous), energy intake (kcal/day: continuous), dietary supplement use (yes, no), education level (high school or less, college or more), marital status (married or cohabitated, unmarried or divorced or widowed), time since surgery (6 month - 1 year, 1 year - 5 years, $\geq 5$ years), stage (II or III), and center

exercise training can reduce fatigue and improve the QOL of cancer patients and survivors [47]. Doing exercise on cancer patients or survivors could improve in their functional capacity and increase tolerance to physical fatigue and metabolic efficiency [48]. Improved metabolic efficiency can change characteristics of skeletalmuscle, and increase the proportion of oxidative fibers or decrease the proportion of glycolytic fibers [49]. Oxidative fibers can remove lactate from blood and they are less fatigable. Therefore, increased muscle efficiency explains how patients with higher physical activity can carry out normal daily activities with less fatigue [47].

Our study had several limitations. First, our study design did not enable us to determine casual direction because we did not measure HRQOL levels after physical activity assessment. Further prospective studies are needed to evaluate changes in HRQOL over time according to physical activity. Secondly, we did not have information on pre-diagnostic levels of physical activity, and our sample size was small. However, given that no 
prospective cohort studies have been conducted on physical activity and HRQOL or mortality in Korea, to our knowledge, this study provides evidence demonstrating the importance of physical activity on Korean breast cancer survivors. Thirdly, we cannot rule out that possibility of information bias by interviewer or interviewee because both physical activity and HRQOL were assessed at the same time. Also, misclassification of physical activity or HRQOL could be introduced. However, given that MET-hour per week of physical activity was well-correlated to physical functioning of quality of life, ranking of physical activity levels might be captured in our study. Lastly, the sampling for this study was not random, which limits our ability to generalize our results to all breast cancer survivors in Korea. However, because the hospitals in our study are major hospitals in metro areas, attracting patients from all over the country, our results may not be confined to narrow scope of subjects. Also, the positive association between physical activity and better quality of life that we observed may not be limited to our study population because of its potential biological and psychological basis.

\section{Conclusion}

In conclusion, engagement in physical activity was related to less fatigue and pain and better sexual functioning among Korean breast cancer survivors. Our findings may warrant further prospective and intervention studies to support the benefit of physical activity in improving the quality of life and survival of Korean breast cancer survivors.

\section{Additional file}

Additional file 1: Table S1. Health-related quality of life (HRQOL) scores according to physical activity levels among breast cancer survivors by body mass index. Table S2. Health-related quality of life (HRQOL) scores according to physical activity levels among breast cancer survivors by menopausal status at the diagnosis. Table S3. Health-related quality of life (HRQOL) scores according to physical activity levels among breast cancer survivors by time since surgery. Table S4. Health-related quality of life (HRQOL) scores according to physical activity levels among breast cancer survivors by age at diagnosis. (DOCX 48 kb)

\section{Abbreviations}

AJCC: American Joint Committee on Cancer; BEAT Cancer: Better Exercise Adherence after Treatment for Cancer; ENERGY: Exercise and Nutrition Enhance Recovery and Good Health for You; EORTC: European Organization for Research and Treatment of Cancer; HEAL: Health Eating Activity and Lifestyle; HRQOL: Health-related quality of life; LS: Least square;

MET: Metabolic equivalent; QLQ-BR23: Quality of Life Questionnaire Breast Cancer Module 23; QLQ-C30: Quality of Life Questionnaire Core 30

\section{Acknowledgements}

We thank the participants and collaborators of this study for their valuable contributions.

\section{Funding}

This work was supported by Research Resettlement Fund for the new faculty of Seoul National University and by the Basic Science Research Program through the National Research Foundation of Korea (NRF) funded by the Ministry of Science, ICT \& Future Planning (NRF-2014R1A2A2A01007794).

Availability of data and materials

Dataset is available on request.

\section{Authors' contributions}

Authors' contributions JL and WS contributed to the conception and design of the study. SJ, EL, ZK, HM, and DN collected the data from the breast cancer survivors at the three hospitals. $J \mathrm{~L}$ and WS analysed and interpreted the data. WS was the major contributor in writing the manuscript. SS, SJ, EL, $\mathrm{ZK}, \mathrm{HM}$, and DN critically revised the manuscript for intellectual content. All authors read and approved the final manuscript.

\section{Ethics approval and consent to participate}

All procedures for this study were approved by the institutional review board of each hospital. Written informed consent was obtained from all participants.

\section{Consent for publication}

No individual's personal data is included.

\section{Competing interests}

The authors declare that there are no conflicts of interest.

\section{Publisher's Note}

Springer Nature remains neutral with regard to jurisdictional claims in published maps and institutional affiliations.

\section{Author details}

${ }^{1}$ Research Institute of Human Ecology, Seoul National University, Seoul 08826, South Korea. ${ }^{2}$ Department of Food and Nutrition, Sookmyung Women's University, Seoul 04310, South Korea. ${ }^{3}$ Breast Cancer Center National Cancer Center, Goyang 10408, South Korea. ${ }^{4}$ Department of Surgery, Soonchunhyang University Bucheon Hospital, Bucheon 14584, South Korea. ${ }^{5}$ Department of Surgery and Cancer Research Institute, Seoul National University College of Medicine, Seoul 03080, South Korea. ${ }^{6}$ Department of Food and Nutrition, Seoul National University, Seoul 08826, South Korea.

Received: 13 February 2017 Accepted: 20 June 2017

Published online: 30 June 2017

\section{References}

1. Ferlay J, Soerjomataram I, Dikshit R, Eser S, Mathers C, Rebelo M, et al. Cancer incidence and mortality worldwide: sources, methods and major patterns in GLOBOCAN 2012. Int J Cancer. 2015;136:e359-86.

2. Jung K-W, Won Y-J, Kong H-J, Oh C-M, Cho H, Lee DH, et al. Cancer statistics in Korea: incidence, mortality, survival, and prevalence in 2012. Cancer Res Treat. 2015:47:127-41.

3. Bower JE, Ganz PA, Desmond KA, Bernaards C, Rowland JH, Meyerowitz BE, et al. Fatigue in long-term breast carcinoma survivors. Cancer. 2006;106: $751-8$.

4. Lawrence DP, Kupelnick B, Miller K, Devine D, Lau J. Evidence report on the occurrence, assessment, and treatment of fatigue in cancer patients. J Natl Cancer Inst Monogr. 2004;32:40-50.

5. Erickson VS, Pearson ML, Ganz PA, Adams J, Kahn KL. Arm edema in breast cancer patients. J Natl Cancer Inst. 2001;93:96-111.

6. Ganz PA, Desmond KA, Leedham B, Rowland JH, Meyerowitz BE, Belin TR Quality of life in long-term, disease-free survivors of breast cancer: a followup study. J Natl Cancer Inst. 2002;94:39-49.

7. Ganz PA, Rowland JH, Desmond K, Meyerowitz BE, Wyatt GE. Life after breast cancer: understanding women's health-related quality of life and sexual functioning. J Clin Oncol. 1998;16:501-14.

8. Kornblith AB, Herndon JE, Weiss RB, Zhang C, Zuckerman EL, Rosenberg S, et al. Long-term adjustment of survivors of early-stage breast carcinoma, 20 years after adjuvant chemotherapy. Cancer. 2003;98:679-89.

9. Ganz PA, Kwan L, Stanton AL, Krupnick JL, Rowland JH, Meyerowitz BE, et al. Quality of life at the end of primary treatment of breast cancer: first results from the moving beyond cancer randomized trial. J Natl Cancer Inst. 2004; 96:376-87. 
10. Wood AJ, Shapiro CL, Recht A. Side effects of adjuvant treatment of breast cancer. New Engl J Med. 2001;344:1997-2008.

11. Brown JC, Huedo-Medina TB, Pescatello LS, Pescatello SM, Ferrer RA, Johnson BT. Efficacy of exercise interventions in modulating cancer-related fatigue among adult cancer survivors: a meta-analysis. Cancer Epidemiol Biomark Prev. 2011;20:123-33.

12. Szymlek-Gay EA, Richards R, Egan R. Physical activity among cancer survivors: a literature review. NZ Med J. 2011:124:1-13.

13. Meneses-Echávez JF, Gonzalez-Jimenez E, Ramírez-Vélez R. Supervised exercise reduces cancer-related fatigue: a systematic review. J Physiother. 2015;61:3-9.

14. Meneses-Echávez JF, González-Jiménez E, Ramírez-Vélez R. Effects of supervised exercise on cancer-related fatigue in breast cancer survivors: a systematic review and meta-analysis. BMC Cancer. 2015;15:77.

15. Irwin ML, Ainsworth BE. Physical activity interventions following cancer diagnosis: methodologic challenges to delivery and assessment. Cancer Investig. 2004;22:30-50.

16. Schwartz AL. Physical activity after a cancer diagnosis: psychosocial outcomes. Cancer Investig. 2004;22:82-92

17. Oldervoll L, Kaasa S, Hjermstad M, Lund J, Loge J. Physical exercise results in the improved subjective well-being of a few or is effective rehabilitation for all cancer patients? Eur J Cancer. 2004;40:951-62.

18. McTiernan A. Physical activity after cancer: physiologic outcomes. Cancer Investig. 2004:22:68-81.

19. Pinto BM, Trunzo JJ. Health behaviors during and after a cancer diagnosis Cancer. 2005:104:2614-23.

20. Schmitz KH, Holtzman J, Courneya KS, Mâsse LC, Duval S, Kane R. Controlled physical activity trials in cancer survivors: a systematic review and metaanalysis. Cancer Epidemiol Biomark Prev. 2005;14:1588-95.

21. McNeely ML, Campbell KL, Rowe BH, Klassen TP, Mackey JR, Courneya KS. Effects of exercise on breast cancer patients and survivors: a systematic review and meta-analysis. Can Med Assoc J. 2006;175:34-41.

22. Robison LL, Demark-Wahnefried W. Cancer survivorship: focusing on future research opportunities. Cancer Epidemiol Biomark Prev. 2011;20:1994-5.

23. Ainsworth BE, Haskell WL, Herrmann SD, Meckes N, Bassett DR Jr, TudorLocke C, et al. 2011 compendium of physical activities: a second update of codes and MET values. Med Sci Sports Exerc. 2011:43:1575-81.

24. Yun YH, Bae SH, Kang IO, Shin KH, Lee R, Im Kwon S, et al. Cross-cultural application of the korean version of the european organization for research and treatment of cancer (EORTC) breast-cancer-specific quality of life questionnaire (EORTC OLO-BR23). Support Care Cancer. 2004:12:441-5.

25. Yun YH, Park YS, Lee ES, Bang S-M, Heo D, Park S, et al. Validation of the Korean version of the EORTC QLQ-C30. Qual Life Res. 2004;13:863-8.

26. Fayers PM, Aaronson NK, Bjordal K, Groenvold M, Curran D, Bottomley A on behalf of the EORTC Quality of Life Group. EORTC QLQ-C30 Scoring Manual. 3rd ed. Brussels: EORTC; 2001.

27. Sprangers M, Groenvold M, Arraras JI, Franklin J, te Velde A, Muller M, et al, The European Organization for Research and Treatment of cancer breast cancer-specific quality-of-life questionnaire module: first results from a three-country field study. J Clin Oncol. 1996;14:2756-68.

28. Montazeri A, Harirchi I, Vahdani M, Khaleghi F, Jarvandi S, Ebrahimi M, et al. The EORTC breast cancer-specific quality of life questionnaire (EORTC QLQBR23): translation and validation study of the Iranian version. Qual Life Res. 2000;9:177-84

29. Alfano CM, Smith AW, Irwin ML, Bowen DJ, Sorensen B, Reeve BB, et al. Physical activity, long-term symptoms, and physical health-related quality of life among breast cancer survivors: a prospective analysis. J Cancer Surviv. 2007;1:116-28.

30. Chen X, Zheng Y, Zheng W, Gu K, Chen Z, Lu W, et al. The effect of regular exercise on quality of life among breast cancer survivors. Am J Epidemiol. 2009;170:854-62

31. Sagen A, Karesen R, Sandvik L, Risberg MA. Changes in arm morbidities and health-related quality of life after breast cancer surgery - a five-year followup study. Acta Oncol. 2009;48:1111-8.

32. Valenti M, Porzio G, Aielli F, Verna L, Cannita K, Manno R, et al. Physical exercise and quality of life in breast cancer survivors. Int J Med Sci. 2008;5:24-8.

33. Rogers LQ, Markwell SJ, Courneya KS, McAuley E, Verhulst S. Physical activity type and intensity among rural breast cancer survivors: patterns and associations with fatigue and depressive symptoms. J Cancer Surviv. 2011;5:54-61.
34. Pinto BM, Trunzo JJ, Reiss P, Shiu SY. Exercise participation after diagnosis of breast cancer: trends and effects on mood and quality of life. Psychooncology. 2002;11:389-400.

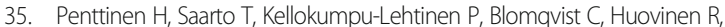
Kautiainen $\mathrm{H}$, et al. Quality of life and physical performance and activity of breast cancer patients after adjuvant treatments. Psycho-Oncology. 2011;20:1211-20.

36. Carayol M, Bernard P, Boiche J, Riou F, Mercier B, Cousson-Gélie F, et al. Psychological effect of exercise in women with breast cancer receiving adjuvant therapy: what is the optimal dose needed? Ann Oncol. 2013;24: 291-300.

37. Rogers LQ, Courneya KS, Anton PM, Hopkins-Price P, Verhulst S, Vicari SK, et al. Effects of the BEAT cancer physical activity behavior change intervention on physical activity, aerobic fitness, and quality of life in breast cancer survivors: a multicenter randomized controlled trial. Breast Cancer Res Treat. 2015:149:109-19.

38. Demark-Wahnefried W, Colditz GA, Rock CL, Sedjo RL, Liu J, Wolin KY, et al. Quality of life outcomes from the exercise and nutrition Enhance Recovery and Good health for you (ENERGY)-randomized weight loss trial among breast cancer survivors. Breast Cancer Res Treat. 2015;154:329-37.

39. Irwin ML, Cartmel B, Harrigan M, Li F, Sanft T, Shockro L, et al. Effect of the LIVESTRONG at the YMCA exercise program on physical activity, fitness, quality of life, and fatigue in cancer survivors. Cancer. 2016:123:1249-58.

40. Hwang JH, Chang HJ, Shim YH, Park WH, Park W, Huh SJ, et al. Effects of supervised exercise therapy in patients receiving radiotherapy for breast cancer. Yonsei Med J. 2008;49:443-50.

41. Kim SH, Shin MS, Lee HS, Lee ES, Ro JS, Kang HS, et al. Randomized pilot test of a simultaneous stage-matched exercise and diet intervention for breast cancer survivors. Oncol Nurs Forum. 2011;38:E97-106.

42. Lee MK, Yun YH, Park H-A, Lee ES, Jung KH, Noh D-Y. A web-based selfmanagement exercise and diet intervention for breast cancer survivors: pilot randomized controlled trial. Int J Nurs Stud. 2014;51:1557-67.

43. Uhm KE, Yoo JS, Chung SH, Lee JD, Lee I, Kim Jl, et al. Effects of exercise intervention in breast cancer patients: is mobile health (mHealth) with pedometer more effective than conventional program using brochure? Breast Cancer Res Treat 2017:161:443-52.

44. Park JH, Lee J, Oh M, Park H, Chae J, Kim DI, et al. The effect of oncologists' exercise recommendations on the level of exercise and quality of life in survivors of breast and colorectal cancer: a randomized controlled trial. Cancer. 2015:121:2740-8.

45. Vallance JK, Courneya KS, Plotnikoff RC, Yasui Y, Mackey JR. Randomized controlled trial of the effects of print materials and step pedometers on physical activity and quality of life in breast cancer survivors. J Clin Oncol. 2007:25:2352-9.

46. Windsor PM, Nicol KF, Potter J. A randomized, controlled trial of aerobic exercise for treatment-related fatigue in men receiving radical external beam radiotherapy for localized prostate carcinoma. Cancer. 2004;101:550-7.

47. Lucia A, Earnest C, Perez M. Cancer-related fatigue: can exercise physiology assist oncologists? Lancet Oncol. 2003;4:616-25.

48. Dimeo F, Rumberger BG, Keul J. Aerobic exercise as therapy for cancer fatigue. Med Sci Sports Exerc. 1998;30:475-8.

49. McArdle WD, Katch Fl, Katch VL. Exercise physiology: nutrition, energy, and human performance. 7th ed. Baltimore, MD: Lippincott Williams \& Wilkins; 2010.

\section{Submit your next manuscript to BioMed Central and we will help you at every step:}

- We accept pre-submission inquiries

- Our selector tool helps you to find the most relevant journal

- We provide round the clock customer support

- Convenient online submission

- Thorough peer review

- Inclusion in PubMed and all major indexing services

- Maximum visibility for your research

Submit your manuscript at www.biomedcentral.com/submit 